\title{
Guest Editorial Foreword
}

\section{Springer Telecommunication Systems: Special Issue on Energy Efficient 5G Wireless Technologies}

\author{
Angelos Antonopoulos • Konstantinos Samdanis • \\ Christos Verikoukis
}

Published online: 20 November 2014

(C) Springer Science+Business Media New York 2014

The vast proliferation of the mobile devices along with the increasing data demands pave the way for the fifth generation $(5 \mathrm{G})$ wireless communication systems, which are envisaged to support significantly higher connection speeds, such as $10 \mathrm{~Gb} / \mathrm{s}$ peak data rates. Heterogeneous networks (HetNets), cloud computing, pervasive networking, Internet of Things (IoT), Machine-to-Machine (M2M) communications, user cooperation and cognitive radio are only some indicative topics that are expected to attract notable research attention in the context of $5 \mathrm{G}$ technologies. However, all these concepts should be studied under a common framework defined by the European Union and the Information and Communications Technology (ICT) industry: energy awareness.

In particular, the development of novel technologies implies higher energy consumption both at the operator's and the end user's side. On the other hand, mobile operators seek the least possible energy consumption to reduce their costs and provide "green" services, while the end users set as high priority the maximization of the battery autonomy. To meet these demands, the energy efficiency should be explicitly considered in the design of new $5 \mathrm{G}$ systems, something

\footnotetext{
A. Antonopoulos $(\varangle) \cdot C$. Verikoukis

Centre Tecnològic de Telecomunicacions de Catalunya (CTTC),

Barcelona, Spain

e-mail: aantonopoulos@cttc.es

C. Verikoukis

e-mail: cveri@cttc.es
}

\author{
A. Antonopoulos \\ K. Samdanis \\ NEC Europe Ltd., Heidelberg, Germany \\ e-mail: samdanis@neclab.eu
}

Technical University of Catalonia (UPC), Barcelona, Spain that will potentially bring changes to the default protocol stack.

This Special Issue of Springer Telecommunications Systems includes energy-oriented multidisciplinary aspects of future networks, ranging from physical layer techniques to communication protocols and network architectures. In response to our call, thirty interesting high-quality research papers have been submitted and, after a rigorous and thorough review process, the top eleven articles have been selected for publication. A summary of the accepted papers is discussed next.

In the first paper, "Exploiting Diversity of Usage to Enhance User Equipment Energy Efficiency in LTE Networks" by Ta and Baras, the authors propose a cooperative framework to improve the lifetime of the mobile terminals in device-to-device (D2D) communications.

In the second paper, "Backhaul-aware Energy Efficient Heterogeneous Networks with Dual Connectivity" by Prasad and Maeder, the authors foster the energy efficient network operation, taking into account the backhaul connections in dense heterogeneous networks.

In the third paper, "A Lifetime Extended Multi-levels Heterogeneous Routing Protocol for Wireless Sensor Networks" by Tyagi et al., the authors focus on the wireless sensor networks that consist of nodes with different energy levels and propose a new routing scheme to increase the network lifetime.

In the fourth paper, "An Energy-Aware Scheme for Efficient Spectrum Utilization in a 5G Mobile Cognitive Radio Network Architecture" by Mavromoustakis et al., the authors propose an energy efficient scheme for the cognitive exploitation of the TV White Space in next generation networks.

In the fifth paper, "Towards Energy-Efficient Cooperative Spectrum Sensing for Cognitive Radio Networks - An 
Overview" by Althunibat et al., the authors provide a survey of the state of the art research in cooperative spectrum sensing for cognitive radio networks and they propose a framework to improve the energy efficiency of such systems.

In the sixth paper, "An Energy Efficient DOA Estimation Algorithm for Uncorrelated and Coherent Signals in Virtual MIMO Systems" by Wan et al., the authors propose an energy efficient scheme for the direction-of-arrival (DOA) estimation of different user equipment terminals by the base station in a parallel way.

In the seventh paper, "Dynamic Two-Stage Beam Training for Energy-Efficient Millimeter-Wave 5G Cellular Systems" by Kim and Hong, the authors introduce an energy efficient protocol for link configuration in millimeter-wave communication, which reduces the number of training signal transmissions in the network.

In the eighth paper, "Energy Efficient Load Balancing for Future Self-Organized Shared Networks" by Monteiro et al., the authors present a novel load balancing scheme that enhances the energy efficiency in self-organized networks shared among multiple operators.

In the ninth paper, "Optimized Handover Strategy with Hybrid Satisfaction Mechanisms" by Zorba and Hassanein, the authors propose a novel vertical handover mechanism that jointly considers the provided data rate and the energy consumption in heterogeneous networks.

In the tenth paper, "A Quantitative Analysis of the Throughput Gains and the Energy Efficiency of MultiRadio Transmission Diversity in Dense Access Networks" by Koudouridis et al., the authors investigate the benefits of multi-radio transmission diversity in dense networks under different scheduling and resource allocation strategies.

Finally, in the last paper, "Relay Selection for Secure 5G Green Communications" by Nomikos et al., the authors deal with application-oriented secure relay selection policies and they propose two schemes for delay-critical applications and energy-efficient communications, respectively.

We would like to thank all the authors for submitting their work in this special issue and the volunteer reviewers for their effort and time during the whole review process. Finally, we would like to express our gratitude to the Editors-in-Chief, Prof. Gavish and Prof. Khan, for their guidance and Mr. Levine for his administrative support.

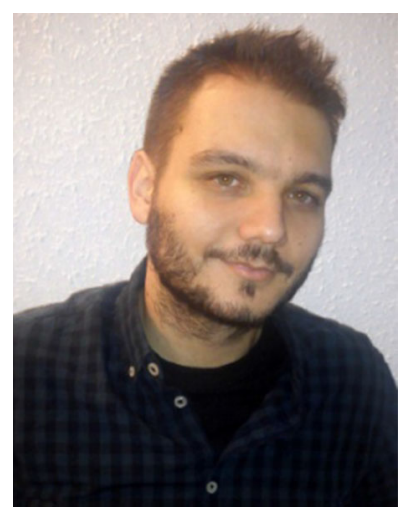

Angelos Antonopoulos received the M.Eng. degree from the Information and Communication Systems Engineering Department, University of the Aegean, Samos, Greece, in 2007 and the Ph.D. degree (cum laude) from the Signal Theory and Communications Department, Technical University of Catalonia, Barcelona, Spain, in 2012. Since January 2013, he has been a visiting Postdoctoral Researcher with Smart Energy Efficient Communication Technologies (SMARTECH), Telecommunications Technological Centre of Catalonia. He has participated in several European and Spanish national projects (e.g., GREENET, Green-T, CO2GREEN, etc.) and has served as an expert evaluator of research projects funded by the Romanian Government through the National Council for Scientific Research. His main research interests include cooperative communications, medium access control protocols, network coding, and energy-efficient network planning. Dr. Antonopoulos has been granted three annual scholarships by the Greek State Scholarships Foundation (IKY), and recently, he received the First Polytechnic Graduates Prize from the Technical Chamber of Greece (TEE-TGC) and the 2014 IEEE GLOBECOM Best 50 Papers Award.

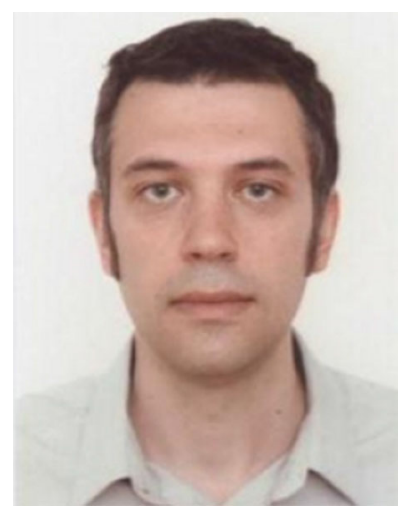

Konstantinos Samdanis (Konstantinos.Samdanis@neclab.eu) received his Ph.D. and M.Sc. degrees in mobile communications from Kings College London. He is a senior researcher and a backhaul standardization specialist with NEC Europe, Germany. He is leading a research project on Long Term Evolution (LTE ) in the field of network management and multi-tenancy, while at the same time, he is involved in the ITN CROSSFIRE project as a principal investigator. He is currently active in $5 \mathrm{GPPP}$, while in the past participated in the iJOIN and BeFemto FP7 projects as well as in IST EVEREST project. Dr. Samdanis is also contributing at the Broadband Forum in the area of Software-Defined Networking/Network Functions Virtualization and is the editor of the TR-293 "Energy-Efficient Mobile Backhaul". In the past he contributed at 3GPP SA5 in the area of energy saving management and conducted a series of tutorials on energy efficiency and green communications at various IEEE conferences including VTC, CCNC, OnlineGreenComm and NOMS. He has served as a guest editor of IEEE Communication Magazine and IEEE MMTC E-Letters, as well as a cochair of the Next-Generation Networking Symposium of the IEEE ICC 2014 and a co-chair of the Networking Symposium of EUCNC 2015. 


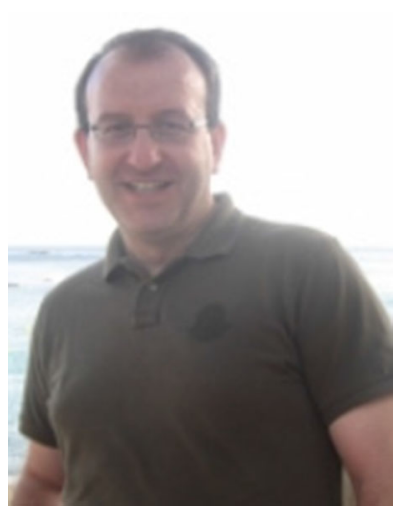

Christos Verikoukis got his Ph.D. from the Technical University of Catalonia in 2000 . He is currently a Senior Researcher at CTTC and an adjunct associate professor at UB. His area of expertise is in the design of energy efficient layer 2 protocols and RRM algorithms for short-range wireless cooperative and network coded communications. He has published 70 journal papers and over 130 conference papers. He is also co-author in 2 books, 14 chapters in different books and in 2 patents. He has supervised $15 \mathrm{Ph}$.D. students and 5 Post Docs researchers since 2004. He has participated in more than 20 competitive projects. He has served as coeditor in 5 special issues while he has participated in the organization of several international conferences. He is also a regular reviewer in a number of international journals. He has appointed to serve as a reviewer in FP7 projects funded by the European Commission and as an EU-independent expert acting as an evaluator. He was General Chair of the 17th and 18th IEEE Workshop on Computer-Aided Modeling, Analysis and Design of Communication Links and Networks (CAMAD'12 and IEEE CAMAD'13), and the TPC Co-Chair of the 15th IEEE International Conference on Ehealth Networking, Application \& Services (Healthcom'13). He has also served as a TPC member in numerous of IEEE conferences (e.g., ICC, GLOBECOM, etc.). He is currently officer (Secretary) of the IEEE ComSoc Technical Committee on Communication Systems Integration and Modeling (CSIM). He received the best paper award of the Communication QoS, Reliability \& Modeling Symposium (CQRM) symposium in the IEEE ICC'11 conference and the EURASIP 2013 Best Paper Award for the Journal on Advances in Signal Processing. 